\title{
Adaptação do Questionário de Ruminação e Reflexão (QRR) para estudantes universitários brasileiros
}

\author{
Cristian Zanon \\ Marco Antônio Pereira Teixeira \\ Universidade Federal de Santa Maria
}

\begin{abstract}
RESUMO
A autoconsciência privada, ou a tendência de um indivíduo para refletir sobre seus pensamentos, motivações, sentimentos e outros aspectos privados da experiência pessoal do si mesmo tem sido muito estudada nas últimas décadas. Esta pesquisa teve por objetivo adaptar o Questionário de Ruminação e Reflexão para estudantes universitários brasileiros, obtendo evidências de validade fatorial e convergente. A versão original em inglês foi traduzida e aplicada a 296 universitários. Uma análise de componentes principais indicou a presença de 2 componentes mais relevantes, correspondendo às dimensões de ruminação e reflexão. Os índices de consistência interna obtidos para ambas as escalas foi de 0,87 . Conforme esperado, a ruminação correlacionou-se positivamente com neuroticismo e negativamente com auto-estima. Por sua vez, a reflexão correlacionou-se positivamente com abertura à experiência. Conclui-se que o instrumento adaptado apresenta validade fatorial e convergente satisfatórias (além de excelente fidedignidade), podendo ser utilizado em futuras pesquisas.
\end{abstract}

Palavras-chave: autoconsciência; traço de personalidade; escala; psicometria.

\section{ABSTRACT \\ Adaptation of the Rumination and Reflection Questionnaire (RRQ) to Brazilian university students}

Private self-consciousness, or the tendency of an individual to reflect on his or her thoughts, motivations, feelings, and other private aspects of the self has been extensively studied during the last decades. The aim of this research was to adapt the Rumination and Reflection Questionnaire to Brazilian university students, investigating its factorial and convergent validity. The original English version was translated to Portuguese and applied to 296 university students. The principal components analysis revealed two main components, corresponding to the proposed dimensions of rumination and reflection. Both scales showed a reliability index of 0,87. As expected, rumination correlated positively with neuroticism and negatively with self-esteem, and reflection correlated positively with openness to experience. In conclusion, the results obtained showed that the adapted instrument has satisfactory factorial and convergent validity, (and excelent reliability), suggesting that it can be used in future research.

Keywords: self-consciousness; reflection; rumination; psychometry.

A capacidade de refletir sobre si mesmo, ou autoconsciência, é uma característica peculiar do ser humano. Durante boa parte do tempo, sua atenção está dirigida a aspectos do seu próprio eu, sejam pensamentos ou sentimentos, ou mesmo o próprio corpo, em sua aparência pública ou reações fisiológicas internas. Todas as pessoas refletem de algum modo sobre si mesmas, embora o grau ou freqüência com que se envolvem nessa atividade varie de indivíduo para indivíduo: alguns refletem mais, outros menos; uns tendem a focalizar mais os aspectos agradáveis das experiências pessoais, outros os desagradáveis; alguns passam horas pensando sobre um mesmo evento, outros não. Contudo, apesar de ser este um aspecto central na experiência humana cotidiana, a psicologia passou a interessar-se pelo estudo da autoconsciência de um modo mais sistemático e experimental somente a partir da década de 1970, quando 
estudos empíricos indicaram que as diferenças individuais no modo de dirigir a atenção sobre si mesmo estavam relacionadas a diversas características comportamentais dos indivíduos (Singer \& Bonanno, 1990).

Um dos trabalhos pioneiros que impulsionaram a pesquisa sobre o tema da autoconsciência foi a construção da Escala de Autoconsciência (EAC) de Fenigstein, Scheier e Buss (1975). Estes autores partiram de uma definição de autoconsciência como traço de personalidade, ou seja, uma disposição razoavelmente estável e trans-situacional que os indivíduos teriam, em maior ou menor grau, a dirigir o foco da atenção sobre si mesmos. Usando uma abordagem empírica de elaboração de instrumentos e técnicas de análise fatorial, os autores identificaram três dimensões subjacentes ao conceito de autoconsciência: a autoconsciência pública (APU), a autoconsciência privada (APR) e a ansiedade social (ANS). A APU refere-se a uma auto-reflexão que leva em consideração o modo como os outros percebem o próprio indivíduo, ou seja, uma consciência de si voltada à imagem pública do sujeito. Já a APR está relacionada à tendência do sujeito a dirigir a atenção a seus pensamentos, sentimentos e fantasias, ou seja, aspectos de si mesmo que não são acessíveis diretamente pelos outros. Por sua vez, a ANS está relacionada com o efeito que a atenção focalizada no próprio eu produz quando na presença de outras pessoas (Fenigstein e cols., 1975).

Desde que foi proposto, o modelo de autoconsciência elaborado por Fenigstein e cols. (1975), especialmente a distinção pública-privada, vem servindo de referência para um grande número de pesquisas na área. $\mathrm{O}$ potencial heurístico da proposta de Fenigstein e colaboradores se verifica pelo interesse que a EAC despertou no meio acadêmico, tendo sido traduzida e adaptada para diversos idiomas como alemão, chinês, espanhol, francês, holandês, italiano, sueco e até mesmo português (Baños \& Belloch, 1990a; Comunian, 1994; Heinemann, 1979; Nystedt \& Smari, 1989; Rimé \& Le Bon, 1984; Shek 1994; Teixeira \& Gomes, 1995; Vleeming \& Engelse, 1981).

O papel da APR no funcionamento psicológico, em especial, é um tópico que tem recebido destaque na literatura. Em uma das primeiras pesquisas que se buscou identificar correlatos da autoconsciência, Turner, Scheier, Carver e Ickes (1978) verificaram que a APR mostrou-se positivamente associada a pensamento reflexivo, auto-monitoração e emocionalidade, e negativamente com auto-estima. Outros estudos sugeriram também associações da APR com neuroticismo (Trapnell \& Campbell, 1999), baixa clareza de autoconceito (Campbell e cols., 1996) e alguns indi- cadores de psicopatologia (Jostes, Pook \& Florin, 1999). De fato, a literatura sugere que a focalização da atenção sobre o self parece estar associada a aspectos negativos do funcionamento psicológico, especialmente a depressão (Baños \& Belloch, 1990b; Ingram, 1990). No entanto, nem sempre as pesquisas que utilizaram a medida de APR proposta por Fenigstein e cols. (1975) encontraram associação entre APR e psicopatologia ou outros indicadores psicológicos negativos (por exemplo, Baños, Belloch \& Perpiña, 1990).

As controvérsias nos achados podem ser atribuídas, ao menos em parte, a divergências quanto à dimensionalidade do construto de APR no instrumento de Fenigstein e cols. (1975) ou em sua versão revisada (Scheier \& Carver, 1985). Burnkrant e Page (1984) foram os primeiros a questionar a unidimensionalidade da APR. Estes autores propuseram e verificaram empiricamente, através de análises confirmatórias, duas subdimensões subjacentes a APR: auto-reflexão (AR) e consciência de estados internos (CEI). A AR refere-se à tendência a pensar sobre si mesmo, enquanto a CEI está relacionada à consciência de sentimentos ou sensações físicas. De um modo geral, os resultados de outros estudos tenderam a confirmar essa diferenciação, embora nem sempre os itens que compuseram uma e outra escala tenham sido exatamente iguais (por exemplo, Cramer, 2000; Lindwall, 2004; Martin \& Debus, 1999; Mittal \& Balasubramanian, 1987). A distinção entre AR e CEI, no entanto, mostrou-se útil pois permitiu identificar efeitos específicos de cada uma dessas dimensões. De fato, resultados de pesquisas mostram que a dimensão autoreflexão se associa mais claramente com efeitos psicológicos negativos (como depressão e baixa autoestima, por exemplo), enquanto a consciência de estados internos não apresenta esse tipo de relação (Campbell e cols., 1996; Conway \& Giannopoulos, 1993; Ghorbani, Watson, Krauss, Davison \& Bing, 2004; Ruipérez \& Belloch, 2003).

Em síntese, os resultados discrepantes que têm sido obtidos com a escala de APR indicam que esse instrumento apresenta imprecisão na operacionalização do construto, o que pode ser até mesmo atribuído à forma como estão redigidos os itens (Ben-Artzi, 2003). Além disso, como sugerem Trapnell e Campbell (1999), a EAC de Fenigstein e cols. (1975) não diferencia os possíveis aspectos motivacionais que podem estar envolvidos na autoconsciência. Segundo os autores, a disposição a focalizar a atenção sobre si mesmo pode ser motivada tanto por necessidades de caráter mais neurótico (como a ansiedade), quanto por necessidades mais puramente epistêmicas (como a curiosidade). 
Em virtude disso, Trapnell e Campbell (1999) propuseram uma nova distinção no âmbito da consciência privada, identificando dois possíveis aspectos no seu funcionamento: ruminação e reflexão. A ruminação seria uma tendência a focalizar e sustentar a atenção, de modo automático, em sentimentos ou eventos considerados desagradáveis ou ameaçadores ao próprio self. Por outro lado, a reflexão seria um processo de pensar sobre si mesmo com características mais epistêmicas, envolvendo uma auto-exploração criativa motivada por um interesse em ampliar o autoconhecimento. Trapnell e Campbell (1999) postularam ainda que a ruminação estaria mais diretamente associada ao traço de personalidade neuroticismo, enquanto a reflexão se associaria mais a abertura à experiência (ambos traços de personalidade dentro do modelo dos Cinco Grandes Fatores). Para testar essas hipóteses, os autores construíram um instrumento de auto-relato com 24 itens, chamado Questionário de Ruminação e Reflexão (QRR). Resultados de análises de componentes principais confirmaram empiricamente a distinção das duas dimensões e as correlações com os traços de personalidade observadas foram de acordo com o esperado. É importante ressaltar que a distinção ruminação-reflexão não corresponde exatamente à diferenciação entre AR e CEI da Escala de Autoconsciência de Fenigstein e cols. (1975), embora exista uma certa semelhança entre os conceitos. Conforme Trapnell e Campbell (1999) apontam, o QRR distingue de modo mais claro os aspectos motivacionais envolvidos nos processos da autoconsciência, algo não contemplado na EAC. Assim, os autores propõem que a ruminação, enquanto uma disposição individual, seria um fator de risco para o desajustamento psicológico, ao passo que a reflexão poderia tanto facilitar quanto prejudicar o ajustamento, dependendo do contexto no qual a pessoa está inserida (sujeitos que, através da reflexão, buscam uma expressão mais genuína da sua singularidade podem se sentir mais satisfeitos consigo mesmos em ambientes onde a individualidade é valorizada, mas isso pode ser um problema em contextos nos quais a tradição e a conformidade são tidos como importantes).

Percebe-se, pois, que a consciência de si, principalmente a consciência voltada para os aspectos privados da experiência pessoal, é uma variável importante que parece ter repercussões sobre o bem-estar psicológico. Conforme apontado anteriormente, contudo, esta autoconsciência privada não parece ser um construto unidimensional, podendo englobar diferentes modos de reflexão sobre o si mesmo. Em especial, os estudos sugerem a existência de duas modalidades de pensamento auto-reflexivo distintas empiricamente: uma de caráter mais epistêmico e autocontrolado, que se associa a um melhor conhecimento de si, das motivações e emoções do sujeito (que pode ser chamada de reflexão); e outra de caráter mais negativo e menos autocontrolado, ligada a pensamentos intrusivos que tendem a perseverar no foco da consciência (que pode ser chamada de ruminação).

$\mathrm{O}$ avanço na compreensão do papel que estas diferentes modalidades de auto-reflexão têm para o funcionamento cognitivo e mesmo a saúde psicológica passa pela necessidade de se ter instrumentos válidos e fidedignos capazes de avaliar estas dimensões. Embora a EAC de Fenigstein e cols. (1975), já traduzida para o português, possa ser utilizada para identificar subdimensões dentro da autoconsciência privada, como sugerem os estudos citados, é evidente que ela também apresenta problemas nesse sentido, pois resultados divergentes têm sido obtidos nas pesquisas com a EAC, que nem sempre identificam as mesmas subdimensões. Isso é problemático pois coloca em questão a validade das medidas que estão sendo utilizadas. Cabe salientar, ainda, que a EAC não foi construída originalmente para medir subdimensões dentro da APR. Sendo assim, torna-se importante contar com instrumentos que avaliem com maior clareza aspectos específicos da auto-reflexão privada, como é o caso do Questionário de Ruminação e Reflexão já mencionado anteriormente (QRR; Trapnell \& Campbell, 1999).

Assim, o objetivo desta pesquisa foi traduzir e adaptar o QRR para universitários brasileiros, buscando obter indícios de validade e fidedignidade da versão adaptada, uma vez que não foram localizados instrumentos brasileiros que avaliem os construtos em questão. O instrumento é disponibilizado na internet por um dos autores no endereço <www.paultrapnell. com>, sendo autorizado o uso em pesquisas sem fins comerciais. Mais especificamente, neste estudo, pretendeu-se: a) verificar a validade fatorial do QRR através de análise de componentes principais, sendo esperada a identificação de dois componentes mais relevantes correspondentes a ruminação e reflexão; b) avaliar a validade convergente das subescalas de ruminação e reflexão, ou seja, suas correlações com outras variáveis com as quais estão teoricamente relacionadas (nesse sentido, esperava-se que a ruminação se correlacionasse positivamente com neuroticismo e negativamente com auto-estima, enquanto a reflexão se correlacionasse positivamente com abertura para a experiência, conforme Trapnell e Campbell, 1999); e c) verificar a fidedignidade das escalas através do índice de consistência interna alpha de Cronbach. 


\section{MÉTODO}

\section{Tradução e adaptação dos itens originais}

Inicialmente a escala em inglês foi traduzida o mais literalmente possível para o português pelos pesquisadores, mas buscando manter o significado dos itens originais. Em seguida, recorreu-se a um procedimento de tradução reversa (back translation), no qual uma pessoa proficiente nas línguas inglesa e portuguesa, e que desconhecia os itens originais, realizou uma tradução dos itens em português para o inglês. Posteriormente, três juízes (falantes nativos de inglês) avaliaram a correspondência de significado dos itens originais com os itens revertidos para o inglês. Para todos os itens pelo menos dois juízes consideraram equivalentes os sentidos das versões em inglês, o que sugere a compatibilidade semântica da versão em português com a versão original em inglês. Por fim, os itens em português foram apresentados a quatro estudantes universitários com o intuito de verificar se eles seriam compreendidos conforme o esperado, o que foi satisfatoriamente verificado. Após estes procedimentos, considerou-se a versão em português da escala pronta para ser testada em seus parâmetros psicométricos.

\section{Participantes}

O instrumento traduzido foi aplicado a 296 estudantes universitários $(64,2 \%$ mulheres) com média de idade de 21,3 anos $(D P=4,3)$, dos cursos de psicologia, educação física, educação especial, pedagogia, matemática, geografia, comunicação social, educação física e engenharia mecânica. Os cursos foram escolhidos por conveniência e a participação dos estudantes no estudo foi voluntária.

\section{Instrumentos}

Foi utilizado um questionário em que, além de informações relativas a sexo e idade, avaliaram-se também as seguintes variáveis:

Ruminação e Reflexão: estas variáveis foram avaliadas através da versão traduzida do Questionário de Ruminação e Reflexão de Trapnell e Campbell (1999), sendo a verificação de sua validade e fidedignidade o objetivo principal desta pesquisa. Os itens são descritos na Tabela 1. A chave de respostas utilizada para cada item foi um sistema Likert de 5 pontos: 1 (discordo totalmente), 2 (discordo), 3 (neutro), 4 (concordo) e 5 (concordo totalmente). Os itens 6, 9, $10,13,14,17,20$ e 24 tiveram suas respostas invertidas previamente, uma vez que possuem sentido con- trário ao dos demais itens das dimensões a que pertencem. São exemplos de itens com sentido revertido: "Eu não perco tempo repensando coisas que já estão feitas e acabadas" (ruminação) e "Eu realmente não sou um tipo meditativo de pessoa" (reflexão).

Auto-estima: foi avaliada através de Escala de Auto-estima de Rosenberg, adaptada ao português por Hutz (2000). Exemplos de itens desta escala são "Levando tudo em conta, eu acho que eu sou um fracasso" (sentido reverso) e "Eu acho que eu tenho tanto valor quanto as outras pessoas". A consistência interna no presente estudo foi elevada (alpha de Cronbach $=0,83$ ).

Neuroticismo e Abertura para Experiência: foram utilizadas duas subescalas de avaliação de traços de personalidade adaptadas para o português que seguem o modelo dos Cinco Grandes Fatores de personalidade (Hutz e cols., 1998). A subescala de neuroticismo é composta de 12 adjetivos como "insegura" e "aborrecida", enquanto a de abertura para experiência contém 12 adjetivos como "audaciosa" e "corajosa". Cada adjetivo é avaliado pelo sujeito em uma escala Likert de 7 pontos. No estudo citado de Hutz e cols. (1998), as escalas de neuroticismo e abertura para a experiência apresentaram índices de consistência interna de 0,80 e 0,78 , respectivamente (além de evidências de validade fatorial). No presente estudo, uma análise de componentes principais prévia dos itens envolvidos, extraindo dois componentes, sugeriu a retirada de alguns deles por terem cargas inferiores a 0,35 no componente esperado. Assim, a escala de neuroticismo ficou composta por 10 adjetivos, e a de abertura à experiência por 11 . Os índices de consistência interna observados neste estudo (alpha de Cronbach) foram 0,81 e 0,75, respectivamente, para neuroticismo e abertura à experiência.

\section{Procedimentos}

A aplicação dos instrumentos foi realizada coletivamente em sala de aula. Após ser obtida permissão dos professores, a proposta da pesquisa foi apresentada aos alunos, destacando-se os seus objetivos, o caráter voluntário da participação e a garantia da confidencialidade dos dados. Aqueles que optaram por tomar parte no estudo assinaram um Termo de Consentimento Livre e Esclarecido de participação na pesquisa antes de preencherem o questionário.

\section{Análise dos dados}

A verificação da validade fatorial das escalas traduzidas de reflexão e ruminação foi feita através de 
procedimentos de análise de componentes principais. A fidedignidade das escalas, por sua vez, foi avaliada através do índice de consistência interna alpha de Cronbach. Já a verificação do padrão de inter-relações entre as diversas variáveis envolvidas no estudo foi feita através de correlações de Pearson.

\section{RESULTADOS}

\section{Estrutura componencial do QRR e fidedignidade das escalas}

O conjunto de 24 itens do QRR foi submetido a uma análise de componentes principais para verificar se emergiriam os dois componentes esperados relacionados a reflexão e ruminação. Optou-se, como no estudo original de Trapnell e Campbell (1999) por utilizar uma rotação oblíqua. Os resultados indicaram a presença de dois componentes mais relevantes (pelo teste de scree), que explicaram $42,8 \%$ da variância total (26,3\% o primeiro componente e $16,5 \%$ o segundo). O índice Kayser-Meyer-Olkin observado, que avalia a adequação dos dados para a realização desse tipo de análise, foi 0,89 , que é considerado muito bom. $\mathrm{O}$ teste de esfericidade de Bartlett apresentou resultado significativo $(p<0,001)$, também indicando adequação dos dados para a análise. Por fim, as comunalidades observadas ficaram entre 0,23 e 0,61 . Os resultados da análise de componentes principais são exibidos na Tabela 1 . Como se pode observar, cada um dos itens apresentou carga componencial superior a 0,40 apenas no componente esperado (e inferior a 0,20 no outro componente), o que resultou em uma solução clara e coerente com as expectativas teóricas. Além disso, as subescalas de reflexão e ruminação apresentaram excelente consistência interna, tendo-se obtido índices alpha de Cronbach de 0,87 para cada uma delas. Análises parciais realizadas para cada sexo separadamente mostraram resultados muito similares.

Tabela 1: Carga Componencial dos Itens nos Componentes I (Ruminação) e II (Reflexão)

\begin{tabular}{|c|c|c|}
\hline \multirow[t]{2}{*}{ ITENS } & \multicolumn{2}{|c|}{ Componentes } \\
\hline & I & II \\
\hline \multicolumn{3}{|l|}{ Ruminação } \\
\hline 1. Minha atenção é freqüentemente focada em aspectos de mim mesmo sobre os quais eu gostaria de parar de pensar. & ,45 & \\
\hline 2. Eu sempre pareço estar remoendo, em minha mente, coisas recentes que eu disse ou fiz. & 66 & \\
\hline 3. Às vezes, é difícil para mim parar de pensar sobre mim mesmo. & ,49 & \\
\hline 4. Muito depois de uma discordância ou discussão ter acabado, meus pensamentos continuam voltados para o que aconteceu. & 68 & \\
\hline 5. Eu tendo a ruminar ou deter-me sobre coisas que acontecem comigo por um longo período depois. & ,75 & \\
\hline 6. Eu não perco tempo repensando coisas que já estão feitas e acabadas." & ,67 & \\
\hline 7. Eu freqüentemente fico revendo em minha mente o modo como eu agi em uma situação passada. & ,75 & \\
\hline 8. Eu freqüentemente me pego reavaliando alguma coisa que já fiz. & ,70 & \\
\hline 9. Eu nunca fico ruminando ou pensando sobre mim mesmo por muito tempo. ${ }^{*}$ & ,49 & \\
\hline 10. É fácil para mim afastar pensamentos indesejados da minha mente. ${ }^{*}$ &, 54 & \\
\hline 11. Eu freqüentemente fico pensando em episódios da minha vida sobre os quais eu não devia mais me preocupar. & ,75 & \\
\hline 12. Eu passo um bom tempo lembrando momentos constrangedores ou frustrantes pelos quais passei. & ,76 & \\
\hline \multicolumn{3}{|l|}{ Reflexão } \\
\hline 13. Coisas filosóficas ou abstratas não me atraem muito. * & & ,74 \\
\hline 14. Eu realmente não sou um tipo meditativo de pessoa. * & & 65 \\
\hline 15. Eu gosto de explorar meu interior. & & ,77 \\
\hline 16. Minhas atitudes sobre as coisas fascinam-me. & &, 53 \\
\hline 17. Eu realmente não gosto de coisas introspectivas ou auto reflexivas. ${ }^{*}$ & &, 65 \\
\hline 18. Eu gosto de analisar por que eu faço as coisas. & &, 58 \\
\hline 19. As pessoas freqüentemente dizem que eu sou um tipo de pessoa introspectiva, "profunda". & &, 58 \\
\hline 20. Eu não me preocupo em auto analisar-me. ${ }^{*}$ & & ,48 \\
\hline 21. Eu sou uma pessoa muito auto investigadora por natureza. & &, 55 \\
\hline 22. Eu gosto de meditar sobre a natureza e o significado das coisas. & & ,71 \\
\hline 23. Eu freqüentemente gosto de ficar filosofando sobre minha vida. & & ,77 \\
\hline 24. Não acho graça em ficar pensando sobre mim mesmo. * & & ,64 \\
\hline
\end{tabular}

Nota. São exibidas apenas as cargas de valor superior a 0,20 .

"Item revertido 


\section{Evidências de validade convergente e diferenças entre os sexos}

A Tabela 2 mostra os resultados da análise correlacional. A ruminação correlacionou-se positivamente com neuroticismo e negativamente com auto-estima. Por sua vez, a reflexão correlacionou-se positivamente com abertura à experiência. Uma correlação positiva foi ainda observada entre ruminação e reflexão. Notese também que a reflexão não apresentou correlação alguma com auto-estima.

Foram calculadas ainda médias de ruminação e reflexão para homens e mulheres. A média das respostas dadas aos itens de 1 a 12 compuseram o escore de ruminação dos sujeitos, e aos itens de 13 a 24 o de reflexão (considerando-se a amplitude dos itens, ou seja, de 1 a 5 ). A diferença observada entre os níveis de ruminação de homens $(M=3,33 ; D P=0,74)$ e mulheres $(M=3,44 ; D P=0,73)$ não se mostrou estatisticamente significativa $[t(294)=1,21 ; p=0,23]$. Porém, a diferença entre as médias de reflexão de homens $(M=3,30 ; D P=0,77)$ e mulheres $(M=3,53 ; D P=0,63)$ foi significativa $[t(182,1)=2,73 ; p=0,01]$, com as mulheres apresentando escores mais elevados.

Tabela 2: Correlações entre Ruminação e Reflexão e demais Variáveis

\begin{tabular}{lcccr}
\hline Variáveis & $(1)$ & $(2)$ & $(3)$ & $(4)$ \\
\hline 1. Ruminação & - & & & \\
2. Reflexão &, $24^{*}$ & - & & \\
3. Neuroticismo &, $39^{*}$ &, 08 & - & \\
4. Abertura à Experiência &,$- 27^{\star}$ &, $29^{*}$ &,$- 27^{*}$ & - \\
5. Auto-Estima &,$- 40^{*}$ &,- 06 &,$- 61^{*}$ &, $30^{*}$ \\
\hline Nota. O zero antes da vírgula foi omitido para facilitar a visualização. \\
${ }^{*} p<0,01$ (correlações de Pearson)
\end{tabular}

\section{DISCUSSÃO}

O objetivo deste estudo foi adaptar o Questionário de Ruminação e Reflexão para ser utilizado com universitários brasileiros. Nesse sentido, os cuidados tomados na fase de tradução do instrumento, com back translation e análise de juízes, conferem uma razoável segurança quanto à compatibilidade semântica entre os itens originais em inglês e os da versão em português.

Por sua vez, as evidências de validade fatorial, obtidas através de análise de componentes principais, mostram que as dimensões de ruminação e reflexão se distinguem empiricamente na versão adaptada do instrumento. A solução componencial encontrada foi clara e distinta, com todos os itens apresentando cargas elevadas (maiores do que 0,40 ) apenas no componente esperado e nenhuma carga superior a 0,20 no outro componente. Estes resultados, além de estarem de acordo com as expectativas, são muito semelhantes aos obtidos com o instrumento original (Trapnell \& Campbell, 1999). Outro resultado altamente satisfatório observado foram os índices de consistência interna (alpha de Cronbach) de 0,87 para ambas as escalas, o que indica que se tratam de escalas de elevada fidedignidade. É importante ressaltar também que as aná- lises de componentes principais realizadas separadamente para homens e mulheres apresentaram resultados muito semelhantes, o que sugere que a distinção entre ruminação e reflexão através do QRR é válida para ambos os sexos.

Os resultados obtidos com as análises correlacionais também se mostraram conforme o esperado. Em primeiro lugar destaca-se a correlação positiva e baixa entre ruminação e reflexão, também observada por Trapnell e Campbell (1999), o que sugere que estas duas disposições, embora possuam algum grau de associação, referem-se a processos distintos de autoconsciência. Ainda, a ruminação correlacionou-se positivamente com neuroticismo e negativamente com auto-estima, o que está de acordo com os achados de Trapnell e Campbell (1999). Tais achados sugerem que a ruminação é uma disposição da autoconsciência associada a dificuldades de ajustamento emocional. Embora não tenha sido levantada nenhuma hipótese específica sobre a relação entre ruminação e abertura à experiência, observou-se uma correlação negativa entre essas variáveis na presente pesquisa. Contudo, no estudo de Trapnell e Campbell (1999), a abertura à experiência medida através de adjetivos não mostrou correlação significativa com a ruminação; já as facetas da abertura denominadas abertura a sentimentos e abertura à fantasia mostraram-se positivamente cor- 
relacionadas com ruminação (magnitudes de 0,24 e 0,22 ). Apenas a faceta chamada abertura a ações mostrou correlação negativa com ruminação $(-0,18)$. É possível que o resultado divergente obtido nesta pesquisa deva-se à forma como foi medida a abertura à experiência. Talvez os adjetivos utilizados estejam mais fortemente associados aos aspectos psicologicamente saudáveis do traço de abertura, o que pode ter influenciado a associação negativa entre ruminação e abertura. Note-se que a correlação positiva observada entre abertura e auto-estima está de acordo com esta interpretação. De qualquer forma, o resultado observado indica que a relação entre ruminação e abertura à experiência é um tópico que precisa ser investigado com mais detalhes em novos estudos.

Por sua vez, conforme esperado, a reflexão correlacionou-se positivamente com abertura à experiência, sendo tal resultado uma evidência de validade convergente da escala de reflexão. É importante observar que a reflexão, embora tenha apresentado associação com a abertura, não se correlacionou positivamente com auto-estima. De fato, como Trapnell e Campbell (1999) sustentam, a reflexão pode tanto ajudar quanto atrapalhar o ajustamento psicológico. Por um lado, sujeitos auto-reflexivos podem ter uma percepção mais acurada de si mesmos e assim buscarem se expressar no mundo de um modo mais singular e autêntico, possivelmente aumentando seu bem-estar psicológico. Por outro lado, uma percepção mais realista de si mesmos pode levar os sujeitos auto-reflexivos a perceberem mais suas limitações e fracassos, tornando-os menos otimistas e felizes (o que é chamado pelos autores de paradoxo da auto-absorção). Portanto, os efeitos que a reflexão pode ter para a saúde psicológica parecem depender do contexto em que o sujeito está inserido e dos valores que ele e sua cultura privilegiam (Trapnell \& Campbell, 1999), constituindo-se em um tema que necessita ser melhor explorado por novas pesquisas.

A diferença entre os sexos observada para a variável reflexão também merece um comentário. No estudo original de Trapnell e Campbell (1999) não apareceram diferenças significativas entre homens e mulheres tanto em reflexão quanto em ruminação. $\mathrm{O}$ resultado observado na presente pesquisa sugere que as mulheres apresentam uma tendência mais meditativa ou auto-exploradora do que os homens. Tal efeito, no entanto, pode ter sido causado não tanto em virtude do sexo, mas de uma possível associação entre sexo e curso universitário peculiar à amostra deste estudo. Cerca de $21 \%$ dos participantes foram estudantes de psicologia do sexo feminino. É possível que estudan- tes de psicologia (independente do sexo) tenham uma disposição à reflexão mais acentuada do que estudantes de outros cursos, sendo essa característica um provável motivador da escolha pelo curso. Assim, a diferença observada neste estudo pode de fato não estar associada ao sexo, e sim a outras características de personalidade. Vale ressaltar que uma análise complementar comparando homens e mulheres apenas do curso de psicologia não mostrou diferença significativa entre os sexos nos escores de reflexão.

Em síntese, pode-se concluir que os objetivos da presente pesquisa, que foram adaptar o QRR para universitários brasileiros e colher evidências de validade e fidedignidade das escalas foi plenamente atingido, estando o instrumento em condições de ser utilizado em novos estudos. Cabe pontuar, no entanto, alguns limites desta pesquisa. Em primeiro lugar, a versão em português foi aplicada apenas em estudantes universitários; portanto, não é possível afirmar que indivíduos com outras características ou outro nível de instrução compreendam e respondam o QRR da mesma forma. Faz-se necessário, portanto, aplicar o instrumento a outros grupos a fim de testar as características do instrumento em outras populações, especialmente amostras clínicas (por exemplo, formadas por sujeitos diagnosticados com depressão - que devem apresentar escores de ruminação mais altos do que grupos de não deprimidos). Em segundo lugar, as variáveis de personalidade foram medidas através de adjetivos marcadores para as dimensões dos Cinco Grandes Fatores de personalidade, e não através de inventários psicometricamente mais sofisticados. Seria importante replicar os achados deste estudo utilizando instrumentos mais completos (medindo facetas de neuroticismo e abertura à experiência, por exemplo), o que não foi feito nesta pesquisa para não estender o tempo de aplicação do questionário (além de não existir, em nosso conhecimento, um inventário validado para medir abertura à experiência disponível em português). Apesar dessas limitações, espera-se que a versão em português do QRR sirva para estimular novas pesquisas no campo da autoconsciência, especialmente sobre o seu papel no funcionamento e no bem-estar psicológico.

\section{REFERÊNCIAS}

Baños, R. M. \& Belloch, A. (1990a). La escala de auto-conciencia (SCS): consideraciones metodológicas y utilidad clínica. Anales de Psiquiatria, 6, 146-152.

Baños, R. M. \& Belloch, A. (1990b). Auto-con(s)ciencia y depression: viajando a través del espejo. Boletin de Psicologia, 26, 7-31. 
Baños, R. M., Belloch, A. \& Perpiña, C. (1990). SelfConsciousness Scale: A study of spanish housewives. Psychological Reports, 66, 771-774.

Ben-Artzi, E. (2003). Factor structure of the private selfconsciousness scale: Role of item wording. Journal of Personality Assessment, 81, 256-264.

Burnkrant, R. E. \& Page, T. J. Jr. (1984). A modification of the Fenigstein, Scheier, and Buss Self-Consciousness Scale. Journal of Personality Assessment, 48, 629-637.

Campbell, J. D., Trapnell, P. D., Heine, S. J., Katz, I. M., Lavalle, L. F. \& Lehman, D. R. (1996). Self-concept clarity: Measurement, personality correlates, and cultural boundaries. Journal of Personality and Social Psychology, 70, 141-156.

Comunian, A. L. (1994). Self-Consciousness Scale dimensions: An iItalian adaptation. Psychological Reports, 74, 483-489.

Conway, M. \& Giannopoulos, C. (1993). Self-esteem and specificity in self-focused attention. The Journal of Social Psychology, 133, 121-123.

Cramer, K. M. (2000). Comparing the relative fit of various factor models of the Self-Consciousness Scale in two independent samples. Journal of Personality Assessment, 75, 295-307.

Fenigstein, A., Scheier, M. F. \& Buss, A. H. (1975). Public and private self-counsciousness: Assessment and theory. Journal of Consulting and Clinical Psychology, 43, 522-527.

Ghorbani, N., Watson, P. T., Krauss, S. W., Davison, H. K. \& Bing, M. N. (2004). Private self-consciousness factors: Relationships with need for cognition, locus of control, and obsessive thinking in Iran and United States. The Journal of Social Psychology, 144, 359-372.

Heinemann, W. (1979). The assessment of private and public selfconsciousness: A german replication. European Journal of Social Psychology, 9, 331-337.

Hutz, C. S. (2000). Adaptação brasileira da escala de auto-estima de Rosenberg. Trabalho não publicado, Universidade Federal do Rio Grande do Sul, Porto Alegre.

Hutz, C. S., Nunes, C. H., Silveira, A. D., Serra, J. G., Anton, M. C. \& Wieczorek, L. S. (1998). O desenvolvimento de marcadores para a avaliação da personalidade no modelo dos cinco grandes fatores. Psicologia: Reflexão e Crítica, 11, 395415.

Ingram, R. (1990). Self-focused attention in clinical disorders: Review and a conceptual model. Psychological Bulletin, 107, 156-176.

Jostes, A., Pook, M. \& Florin, I. (1999). Public and private selfconsciousness as specific psychopathological features. Personality and Individual Differences, 27, 1285-1295.
Lindwall, M. (2004). Factorial structure and invariance across gender of the swedish Self-Consciousness Scale. Journal of Personality Assessment, 82, 233-240.

Martin, A. J. \& Debus, R. L. (1999). Alternative factor structure for the revised Self-Consciousness Scale. Journal of Personality Assessment, 72, 266-281.

Mittal, B. \& Balasubramanian, S. K. (1987). Testing the dimensionality of the Self-Consciousness Scale. Journal of Personality Assessment, 51, 53-68.

Nystedt, L. \& Smari, J. (1989). Assessment of the Fenigstein, Scheier, and Buss Self-Consciousness Scale: A swedish translation. Journal of Personality Assessment, 53, 342-352.

Rimé, B. \& Le Bon, C. (1984). Le concept de conscience de soi et ses opérationnalisations. L'Année Psychologique, 84, 535-553.

Ruipérez, M. A. \& Belloch, A. (2003). Dimensions of the selfcounsciousness scale and their relationship with psychopathological indicators. Personality and Individual Differences, 35, 829-841.

Scheier, M. F. \& Carver, S. C. (1985). The Self-Consciousness Scale: A revised version for use with general populations. Journal of Applied Social Psychology, 15, 687-699.

Sheck, D. T. L. (1994). Assessment of private and public selfconsciousness: A chinese replication. Journal of Clinical Psychology, 50, 341-348.

Singer, J. L. \& Bonanno, G. A. (1990). Personality and private experience individual variations in consciousness and in attention to subjective phenomena. Em Pervin, L.A. (Org.), Handbook of Personality: Theory and Research (pp. 429-431). New York: The Guilford Press.

Teixeira, A. P. M. \& Gomes, W. B. (1995). Self-Consciousness Scale: A brazilian version. Psychological Reports, 77, 423-427.

Trapnell, P. D. \& Campbell, J. D. (1999). Private SelfConsciousness and the Five-Factor Model of personality: Distinguishing rumination from reflection. Journal of Personality and Social Psychology, 76, 284-304.

Turner, R. G., Scheier, M. F., Carver, C. S. \& Ickes, W. (1978) Correlates of self-consciousness. Jounal of Personality Assessment, 42, 285-289.

Vleeming, R. G. \& Engelse, J. A. (1981). Assessment of private and public self-consciousness: A dutch replication. Journal of Personality Assessment, 45, 385-389.

Recebido: 25/04/2006 Revisado: 06/06/2006 Aceito: 05/07/2006

\section{Sobre os autores:}

Cristian Zanon: Psicólogo, formado pela Universidade Federal de Santa Maria.

Marco A. P. Teixeira: Doutor em Psicologia do Desenvolvimento (UFRGS) e Professor Adjunto no Departamento de Psicologia da Universidade Federal de Santa Maria.

Endereço para correspondência: Marco A. P. Teixeira - Rua Floriano Peixoto, 1750 sala 309 - 97015-372 Santa Maria/RS -Endereço eletrônico: mapteixeira@yahoo.com.br 